\title{
Osteoporosis in Populations with High Calcium Intake: Does Phosphate Toxicity Explain the Paradox?
}

\author{
Abbas Ali Mahdi ${ }^{1}$ Ronald B. Brown ${ }^{2} \cdot$ Mohammed S. Razzaque $^{3,4}$
}

Received: 14 August 2015/ Accepted: 1 September 2015/Published online: 25 September 2015

(C) Association of Clinical Biochemists of India 2015

Phosphorus is an essential mineral component of the human body, and is essential for energy metabolism (ATP formation), maintenance of intracellular $\mathrm{pH}$, and cell signaling; it is also an important element of cell membranes, nucleic acids (i.e. DNA and RNA molecules), and the skeletal system. Phosphorous has a very strong electronegative attraction with calcium-having electronegative values on the Pauling scale of 2.1 for phosphorus and 1.0 for calcium. The strength of this attraction may help explain why phosphate toxicity due to dietary phosphorus overload can so easily impact calcium homeostasis (Fig. 1). At physiologic $\mathrm{pH}$ (7.4), extracellular phosphate is mostly present as $\mathrm{Na}_{2} \mathrm{HPO}_{4}$ and $\mathrm{NaH}_{2} \mathrm{PO}_{4}$. Once absorbed, phosphate combines with calcium and accumulates mostly in bone and teeth to form the structural basis and rigidity of these organs.

As calcium is resorbed from bone in response to secondary hyperparathyroidism, gradual loss of bone mass causes osteoporosis. Phosphate toxicity that can induce this disorder of mineral metabolism may help explain the calcium paradox, which is defined by the World Health Organization and Food and Agriculture Organization of the United Nations as the high intake of calcium consumed by

Mohammed S. Razzaque

mrazzaque@forsyth.org; debpur@gmail.com

1 Department of Biochemistry, King George's Medical University, Lucknow, Uttar Pradesh, India

2 College of Human Ecology, Kansas State University, Manhattan, KS, USA

3 Department of Applied Oral Sciences, Forsyth Institute, Harvard School of Dental Medicine Affiliate, Cambridge, MA, USA

4 Division of Research and Development, VPS Health Care, Abu Dhabi, UAE populations in countries with the highest prevalence of osteoporosis [1]. Of relevance, according to the National Osteoporosis Foundation, $50 \%$ of women over the age of 50 and around $12 \%$ of men are likely to have an osteoporosis-related fracture. Cow milk consumed in these countries contains high levels of both calcium and phosphorus. By contrast, human milk contains about six times less phosphorus than cow milk. With a calcium-phosphorus ratio of approximately $1.24: 1$, cow milk alone can never meet the human adult RDA's of $1000-1200 \mathrm{mg}$ calcium and $700 \mathrm{mg}$ phosphorus with a calcium-phosphorus ratio of 1.4:1-1.7:1. In addition, only approximately one-third of the calcium in cow milk is bioavailable in humans [2] while much of the phosphorus is absorbed [3], thus reducing cow milk's bioavailable calcium level to less than half the amount of phosphorous absorbed.

Consumed in large quantities, the high phosphorus content of cow milk intended for calves along with calcium's poor bioavailability in humans has the potential to upset the serum calcium-phosphorus balance in humans, triggering parathyroid hormone to release calcium from bone. The high protein content of large quantities of milk can also contribute to disturbed calcium balance [4]. The overall net result is that the more dairy consumed in combination with other dietary sources of phosphorus and protein, the higher the risk for osteoporosis. In fact, analyzing the diets of 1035 women, Sellmeyer et al. [5] found that women who consumed a high animal-to-vegetable protein ratio had a significantly higher rate of bone loss than those who only consumed vegetable protein; such bone loss led to increased hip fracture by a factor of four times. It is speculated that sulphur-containing amino acids in protein-rich foods, by generating sulfuric acid, can induce acidosis to stimulate osteoclastic activity and promote osteoporosis. Of significance, respiratory or 


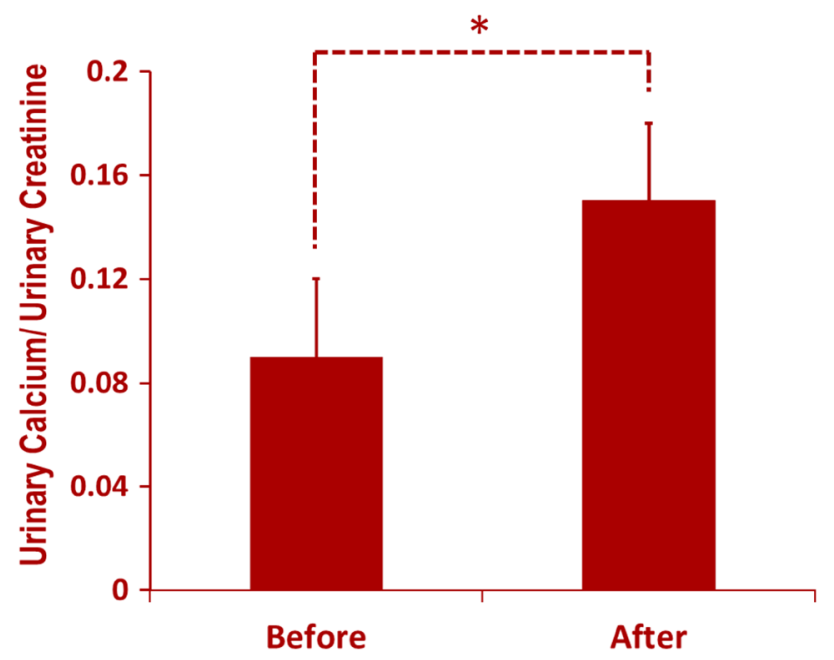

Fig. 1 Effects of phosphate-rich carbonated soda drink on calcium metabolism. The mean level of urinary calcium (adjusted using urinary creatinine) in 35 overnight fasted volunteers (age: $21.2 \pm 0.4$ years old; 13 males, 22 females) before and after drinking carbonated soda. Please note a significant $(* p=0.001)$ increase in urinary calcium excretion $2 \mathrm{~h}$ after consuming $350 \mathrm{ml}$ of carbonated soda, as compared to fasting levels. In contrast, there was no such change in urinary calcium excretion before or after drinking water in 20 control subjects (age: $\mathrm{n}=20$; average age $19.9 \pm 0.3$ years old; 7 males, 13 females; data not shown) $[14,15]$

metabolic acidosis may hydrolyze intracellular organic phosphate-containing compounds and release them into the extracellular compartment to shift the phosphate balance towards hyperphosphatemia, as usually seen in cell lysis disorders such as tumor lysis syndrome, hemolytic anemia, and rhabdomyolysis.

The U.S. Department of Agriculture (USDA) recommends three eight-ounce servings of milk or equivalent dairy products a day. Of relevance, the Indian Council of Medical Research (ICMR) also recommends similar intake of milk or equivalent dairy products per day. Three cups of nonfat milk provides $764 \mathrm{mg}$ of phosphorus or $109 \%$ of the RDA for a 2000 calorie diet, while supplying only $13 \%$ of calories needed for energy. Having reached one's dietary allowance for phosphorus with so few calories provided by three servings of nonfat milk, it is next to impossible to avoid consuming additional phosphorus greatly in excess of the RDA from the remaining $87 \%$ of one's required calorie intake, unless one consumes mostly empty-calorie foods that lack micronutrients. The problem is that the phosphorus caloric density (mg phosphorus per calorie) is very high in nonfat milk.

Generally, the USDA Dietary Guidelines for Americans is incongruous with the latest research on dietary phosphorus toxicity, with little indication for change in the 2015 Dietary Guidelines [6]. For example, a 2000-calorie eating pattern recommended by the MyPlate program of the
USDA Center for Nutrition Policy \& Promotion [7] averages $1884 \mathrm{mg}$ of phosphorus a day, far above the intake level where mortality effects are seen [8]. This problem may be rectified by replacing much of the recommended dairy intake with more green leafy vegetables and other sources of plant-based calcium that have lower absolute amounts of phosphorus and lower phosphorus caloric densities. Countries with higher intake of plant-based foods and lower dairy intake in their non-Westernized traditional diets, like Asian, Middle Eastern, and African countries, have much lower rates of osteoporosis than North American and European countries [9].

In the Harvard Nurses' Health Study, a 12-year prospective study conducted on 77,761 women (age: 34-59 years), those who consumed the highest amount of calcium from dairy foods broke more bones than those who rarely drank milk [10]. Similarly, in the Health Professionals Follow-up Study (HPFS) with 331,234 men (age: 40-75 years), the relative risk of forearm and hip fractures in men was greater among the highest quintile of calcium intake (from foods plus supplements) compared with those in the lowest quintile; such observations were obtained after adjusting for age, smoking status, body mass index (BMI), physical activity, alcohol consumption and total energy intake [11]. Of relevance, compared to American women, Chinese women have much less osteoporosis, which investigators attribute to Chinese women avoiding dairy products and taking most of their calcium from vegetables, despite having similar life expectancies (China 77 years vs. America 81 years; Global Health Observatory Data Repository: Life expectancy, 2013). For example, a cup of cow whole milk weighs $244 \mathrm{~g}$ and provides $276 \mathrm{mg}$ calcium, while $100 \mathrm{~g}$ of raw collard greens with $144 \mathrm{~g}$ of orange weighs as much as a cup of milk and contains $290 \mathrm{mg}$ calcium. Moreover, calcium is twice as bioavailable from dark leafy greens than from milk [2], and the combination of orange and collard greens provide only $45 \mathrm{mg}$ of phosphorus compared to $205 \mathrm{mg}$ of phosphorus in a cup of cow whole milk.

In pregnancy and lactation, the nutrient needs of the growing fetus and infant may increase transient bone mineral losses in mothers from which not all mothers fully recover, despite increased intestinal absorption of calcium and increased calcium intake during pregnancy and lactation [12]. Research needs to investigate the role of phosphate stress from excess dietary phosphorus intake by pregnant and lactating mothers, especially from dairy products that reduce the dietary calcium-phosphorus ratio and which may increase maternal bone loss. Studies have shown that lowering excessive phosphorus intake rather than increasing calcium intake beyond adequate levels is required to properly balance the dietary calcium-phosphorus ratio. Given that osteoporosis and its paradoxical 
association with vascular calcification is noted in numerous human studies, it is highly likely that phosphate burden that promotes loss of mineral from bone can also promote calcification in the vascular wall. Since features of phosphate stress can appear after consumption of a high-phosphate diet, even when serum phosphate levels are within the normal range [13], it is important to ensure normal phosphate balance through adequate dietary intake to maintain calcium-phosphate balance for a healthy life, as its imbalance can have far-reaching and irreversible clinical consequences.

\section{Compliance with Ethical Standards}

Conflict of interest All the authors declare that they have no conflict of interest.

Ethical Approval This article does not contain any studies with human participants or animals performed by any of the authors.

\section{References}

1. WHO/FAO. Chapter 4, calcium. Vitamin and mineral requirements in human nutrition. 2nd ed. Geneva: World Health Organization; 2004.

2. Lanou AJ. Should dairy be recommended as part of a healthy vegetarian diet? Counterpoint. Am J Clin Nutr. 2009;89:1638S$42 \mathrm{~S}$.

3. Giles MM, Fenton MH, Shaw B, Elton RA, Clarke M, Lang M, et al. Sequential calcium and phosphorus balance studies in preterm infants. J Pediatr. 1987;110:591-8.

4. Recker RR, Heaney RP. The effect of milk supplements on calcium metabolism, bone metabolism and calcium balance. Am J Clin Nutr. 1985;41:254-63.
5. Sellmeyer DE, Stone KL, Sebastian A, Cummings SR. A high ratio of dietary animal to vegetable protein increases the rate of bone loss and the risk of fracture in postmenopausal women. Study of Osteoporotic Fractures Research Group. Am J Clin Nutr. 2001;73(1):118-22.

6. National Kidney Foundation. Until phosphorus gets on the USDA's radar labeling policy won't change: 2014.

7. Center for Nutrition Policy and Promotion. MyPlate: sample menus for a 2000 calorie food pattern. 2011. http://www.cnpp. usda.gov/USDAFoodPatterns.

8. Chang AR, Lazo M, Appel LJ, Gutierrez OM, Grams ME. High dietary phosphorus intake is associated with all-cause mortality: results from NHANES III. Am J Clin Nutr. 2014;99(2):320-7.

9. Cauley JA, Chalhoub D, Kassem AM, Fuleihan GE. Geographic and ethnic disparities in osteoporotic fractures. Nat Rev Endocrinol. 2014;10:338-51.

10. Feskanich D, Willett WC, Stampfer MJ, Colditz GA. Milk, dietary calcium, and bone fractures in women: a 12-year prospective study. Am J Public Health. 1997;87(6):992-7.

11. Owusu W, Willett WC, Feskanich D, Ascherio A, Spiegelman D, Colditz GA. Calcium intake and the incidence of forearm and hip fractures among men. J Nutr. 1997;127(9):1782-7.

12. Kalkwarf HJ, Specker BL. Bone mineral changes during pregnancy and lactation. Endocrine. 2002;17:49-53.

13. Osuka S, Razzaque MS. Can features of phosphate toxicity appear in normophosphatemia? J Bone Miner Metab. 2012;30(1):10-8.

14. Shutto Y, Shimada M, Kitajima M, Yamabe H, Saitoh Y, Saitoh $\mathrm{H}$, et al. Inadequate awareness among chronic kidney disease patients regarding food and drinks containing artificially added phosphate. PLoS One. 2013;8(11):e78660.

15. Shutto Y, Shimada M, Kitajima M, Yamabe H, Razzaque MS. Lack of awareness among future medical professionals about the risk of consuming hidden phosphate-containing processed food and drinks. PLoS One. 2011;6(12):e29105. 